\title{
Action attribution in schizophrenia: evidencing neural correlates of reasoning?
}

\author{
Denis Larrivee* \\ Loyola University Chicago, Mind and Brain Institute, University of Navarra, Spain
}

\begin{abstract}
The association between the bodily representation of the individual in neural activity of the brain and the construct of the self appears as a consistent and key relationship in varied self-perceptions. Nonetheless, while its perceptual realization implicates a need for the physical instantiation of the construct, variation in its representational content suggest that there exists a second determinant shaping self-representation. Impairments in action attribution but not in automatic, predictive processing that are observed in schizophrenia suggest that this latter determinant fails to link drive to goal decisional mechanisms with bodily representation. Complementary saliency incentivization mechanisms that enhance goal directed, predictive precision, on the other hand, may function to elicit independence from probabilistic predictions by facilitating rational goal inferencing.
\end{abstract}

\section{Introduction}

Disturbances of self traditionally mark diagnostic evaluation of the schizophrenia patient [1]. An abnormal sense of ownership of the body, loss of ego boundary, and confused sense of self agency comprise several of a variety of phenomenological descriptions that have been used to subjectively characterize the disease [2]. The sense of self expressed through body ownership entails implicit and tacit feelings, both that one's body is the physical reality of one's self and that it is uniquely the personal possession of the self. This sense of identification with and possession of the body is experientially ongoing, as John Locke noted "a thinking intelligent being that has reason and reflection and can consider itself as itself the same thinking thing in different times and places [3]." It can occur, therefore, whether bodily actions are initiated by the self, or whether they are actions that are performed on the body.

It is the capacity to perceive the self in such dual and differing states that distinguishes the notion of the self from the sense of self elicited for action attribution [4]. In this latter sense the self is the source of action initiation, which thus distinguishes the self as a source from which bodily actions emerge. Nonetheless the perceptual realization that the body remains the source of its actions links the perception of self to the body in attributed actions.

The association between a representation of the whole individual by his body in the neural activity of the brain, that is, as a global brain state, appears as a consistent and key relationship in varied perceptual realizations of the self. Infants perseverating toward a hidden object where they have previously reached for the object rather than where they have seen it last hidden, for example, the A not B task, is regarded as a failure of one of a class of executive control tasks [5]. This failure in the inability to move toward a goal where last seen, has been interpreted as a failure in motor planning due to maturational insufficiency in mechanisms needed to situate the motor plan, that are associated with representing the self as the whole body [6]. In like manner, the representation of the body is used to configure the spatial domain that distinguishes self from other in social settings [7]. Finally, the minimal self has been indexed to the heart rate as a crucial index of bodily viability [8].

Conversely, while the varied perceptual realizations of the construct implicate a need to instantiate a bodily representation of the self, the representational variation that characterizes the perceptual constructs suggest that there also exists a second determinant that acts to variably shape the neural representation of the body according to experiential exigencies. As a corollary, psychiatric diseases impacting the self, like schizophrenia, can be expected to have a variable impact on the capacity to link the role of the bodily dynamic to the structuring of the self's representation [9]. The point of a second determinant needed to shape bodily representation and the impact of schizophrenia on this process will be taken up here in the specific case of action attribution. Specifically, it will be argued that this second determinant may relate primitives of rational inferencing to bodily representation, which are revealed in schizophrenia lesioning.

\section{Simulation in motor imagery: eliciting the self in dynamic action}

The modern concept of the neural representation of the self, evoked in circumstances where the body is dynamically engaged in intentional actions, has evolved from several experimental legacies traced to the notion of the motor image [10-12]. This image is so designated to indicate a covert action undertaken only mentally and as a simulation of a non-executed action. In its current understanding the motor image represents the feature elements of an intended motor trajectory, that is, the projected series of motions that will be executed in a motor plan. Hence, it contains the signal features needed for plan execution [4].

These features entail distinct and reciprocal contributions from central and peripheral origins; accordingly, they underscore the essential unity of dynamic performance even in its covert formulation

${ }^{\star}$ Correspondence to: Denis Larrivee, Loyola University Chicago, Mind and Brain Institute, University of Navarra, Spain, E-mail: sallar1@aol.com

Received: July 12, 2018; Accepted: July 24, 2018; Published: July 27, 2018 
[13]. Central influences have been classically demonstrated by the presence of movements undertaken in the absence of sensorial input. Lashley, notably, observed that humans, and animals, were capable of motor actions despite the loss of afferent input.Later experiments in monkeys also confirmed a central origin by showing that those with deafferentation of spinal dorsal motor roots [14], that is, a lesion in the immediate locus of afferent input to the motor neuron junction, could still exhibit pointing movements. How central influences were executed became apparent in studies of consecutive motions, where the original relationship between a movement and its spatiotemporal origin had been altered. In such circumstances limb movements failed to correspond to their expected trajectories, involving instead a mis reaching that was then followed by progressive compensatory movements [15]. This was interpreted to indicate that subsequent movement, undertaken when the respective relative coordinates were altered, responded to a memorized centrally evoked motor command that was sent to the sensory cortex, termed an efference copy or corollary discharge.

In continuous motions, however, sensory cues are essential to motor execution, where both are coupled in a mutually reciprocal and sustained process. This is necessary since as the body undergoes motion, its spatiotemporal position is continually changing; so also, the sensory cues that reference it [16]. This peripheral contribution has been shown to provide an ongoing stream of sensory updating that serves to continually adjust motor execution and that has been likened to a perception action loop. Here resonating, sensorial input streams inwardly, continually modifying executable actions. In effect, this functional synergy constrains both the patterns of information inflow and outflow and so regulates the organism's perception of the external world as well as its interaction with it [17]. The significance of this peripheral and perceptual influence is demonstrably evident in studies investigating the body's perceptual and dynamical interaction with the environment in developing infants, acquired habitual motor abilities, biodynamic studies showing that movement and proprioception are intrinsically related to perception, and in the motoric encoding of actions such as reaching [18-21].

Significantly, the body's topological, three-dimensional configuration is critical for coordinated and unified performance. Intuitively this is evident since the body's perimeter and not the brain's neural architecture is the junction where the individual encounters the world. By situating the point of incidence of sensorial reception to its relative bodily mooring, sensorial input modulates information flow as a function of the body's configuration.

Among the senses somatotopic input is unique for structuring the representation of the body, although other senses also have limited contributions. Existing studies show, for example, that somatotopic afferents are essential to the elicitation of self-identity, a staged process emerging throughout the whole body. Indeed, in the absence of bodily input there would be no percept. Beginning with these afferents a threedimensional postural image is successively built up from segmented zones that are progressively and hierarchically assembled en route to the brain. Much of this assembly appears to occur en route at spinal and brain stem levels. In effect the experiential and dynamical operation of this recurrent framework structures a prototypical platform where peripherally enacted and complex integration increasingly occurs. Interactive events with the body are thereby mapped to their respective bodily locus yielding a temporal evolution of bodily contextualized events. Building on this platform, neural activity can be assimilated from their various sittings to generate a common dynamical representation, that is, a bodily entity of which the representation is indicative [22].

\section{Linking the whole to intentional action}

Insights drawn from the motor image, understood in its compositional formulation, posit that bodily representation is a key feature stabilizing individual motions of the plan as a teleologically situated undertaking; that is, one inscribing actions that link an agent with an objective terminus. The percept of the self is thus not an abstraction but a real image emerging from throughout the body and identifying it with a spatiotemporal reality. Accordingly, it appears to constitute an ontological feature needed to confer individual unity for performative and other ends [23]. Of significance, core notions of the self are also situated in emotive dimensions associated with interoceptive feelings localized to peripheral, somatic loci [24,25]. Hence, the bodily representation is also invested with emotional salience that imbues the self with normative significance.

By building on the unitary dynamism established in reciprocal, body brain exchanges the percept can be evoked to guide unified action when called upon. In developing toddlers, for example, discrepancies between explicit and implicit formats in false-belief tests for navigational affordances reveal an inability to mentally construct a navigational trajectory by 'situating oneself and making moves' of the task at hand, consistent with observations made in the A not B task [6]. In other words, it is necessary to conceive of the self as performing bodily action in order to construct its motor trajectory, an ability absent in very young children.

Here, actions must first be distinguished as one's own before they can be bound to the self representation. In Held's proposal bodily actions are distinguished from a surrounding and mobile terrain by comparing them with their efference copy stored in the sensory cortex. Actions consistent with the planned trajectory can be identified thereby as dynamic events belonging to the body. By then linking motions that have been so identified to a representation of the self it is possible also to ascribe a causal origin to the bodily representation; in other words to implement a mechanistic proposal for self agency [26]. Sensorially, this link appears to be structured primarily by visual input, although, as in the case of bodily representation, other senses also provide limited afferent contribution. The use of different sensory modalities means, by extension, that the processes associating executed actions with the body are sensorially distinguished from those structuring the representational content of the body. Intuitively, this can be understood as appropriating adequate sensory performance for mobile versus stable objects that are ontologically distinguished as part to whole in their dynamic realization. This link between the actions and their bodily association, dubbed the Comparator Model by Frith [27], proposes that inscribed in the representational content of the motor image is a sense of agency that is linked with the representation of the body. This is also to say that a critical feature of self-agency is its holistic character that, accordingly, needs to be systemically distinguished.

Consistent with this interpretation, recent studies reveal that physical constraints limiting the range of activities that may be undertaken by the self are systemically imposed [28], that is, they are defined in terms of their systemic realization. Accordingly, systemic activities associated with self-agency evoke not just executive and motor neural circuits but entail body wide internal constraints that inform and integrate goal oriented performance. Purposeful behaviors thus link the representational content of the self to the pursuit of organismal and environmental interactions. In these studies constraints placed on these behaviors are due to a need to use free energy efficiently, which necessarily constrains the potential range of actions that the whole body engages in. 
Accordingly, strategical motor planning necessitates inferences about actions that integrate performance and maximize energy efficiency, which means that such inferences must be made in the context of the self as represented by the body. From these, and other experimental findings it is intuitive to see why the observed events and processes hypothesized by Von Holst et al. [11] and by Frith et al. [26] require a 'predictive processing' to engage motion. Predictions are needed if one is to engage in actions, that is, actions that are intended to be carried out by the self and are not merely passive responses to external events. Since all external contingencies cannot be known beforehand, neither can all energetic consequences of the intended actions. The expectation of the action, its prediction, affords a first approximation open to correction that can structure the sequence that follows, as goal directed and energetically consilient.

\section{Schizophrenia: pathological influences on the bodily representation of self}

Disturbances in the sense of self that mark schizophrenia in prodromal and acute stages have led to the recognition of the loss of self as a core symptom [29]. The consensus on its loss is based on neurocognitive and psychopathological evidence, where both body ownership and sense of agency are impacted, with affected individuals exhibiting distinct and substantive physical and neural signatures [30].

These physical signatures have been shown to extend to effective connectivities based on methodological approaches using imaging modalities [31]. In normal patients, notably, there is a consistently high correspondence between fMRI modules and the modules of structural networks from diffusion imaging, across a wide range of populations. In patients with child onset schizophrenia, by contrast, there is a decrease in modularity and there are significantly different modular communities between affected and non-affected individuals, particularly in the right insular and perisylvian regions [32]. That is, information exchange both within modules and interareally between modules appears to be affected.

Given that these and other physical alterations are consistently observed, this raises questions of whether and how representational content of the self may be affected and how this may be linked to the body. Insight into the neural features that these results may implicate can be inferred from misattribution errors that are experimentally evoked in normal individuals and that appear to be pathologically exacerbated in shizophrenic individuals. In such experiments uncertainty about bodily motion can be artificially induced, for example, by preventing direct visual control of the motion and substituting an 'alien' hand to perform similar movements. In many cases the individual misattributes the motion of the alien hand to himself [33]. Here the link between seeing and attribution is broken, suggesting the implementation of visual sensoria in linking movements to a source identified with the self. On the basis of such experimental results, Feinberg [34] and later Frith [35] proposed that deficient self-monitoring is greatly exaggerated in the schizophrenic patient.

Yet, the association of the motions with a physical representation of the self, as pointed out by Jeannerod [9], is processionally complex, and multiply articulated, includes single appendage motions, that are linked to efference copy transmission, predictive processing which entails automated inferences of whole body performance, and a conscious sense of agency that links individual motions to a selfrepresentation that emanates from the whole body. How attribution is impaired is therefore unclear in these earlier proposals.
Based on evidence from schizophrenic patients Jeannerod has concluded that there exist two levels of self-recognition, one automatic for action identification, the second, conscious and employed for conducting intentional actions. These patients fall within a class where intentional actions but not automatic attributable actions are affected, that is the etiological basis of the disease is associated with an inability to attribute intentional actions to the self-representation.

\section{Action attribution: freeing bodily representation from energy contingencies}

However, since the whole body is used to anchor self-representation, both in automatic predictive processing circumstances and in intentional goal directed ones, the question of how the representational content of the whole body is distinguished for use in either mode is raised. Schizophrenia patients of the class studied by Jeannerod, notably, automatically adapt their movements to visuomotor conflicts, reaching their targets despite distorted visual feedback. That is, they appear to be unimpaired in automatically making needed predictions about the efficacy of their performance, incorporating them in efficient and coordinated motor strategies. Their automatic actions thus appear to successfully negotiate the needed inferential terrain; that is, understood in a Bayesian sense, where they are necessarily contextualized to the whole body [28].

On the other hand, schizophrenia patients are consistently worse than normal individuals in judging whether movements in attribution paradigms are their own or belong to another. In patients with first rank symptoms, that is, with symptoms most indicative of the disease, attribution errors amounted to nearly $80 \%$ of all detected, compared to $30 \%$ for normal individuals. In other words, schizophrenia patients appear to lack a capacity to consciously associate their self-initiated motions to a representation of the self. As the whole body representation itself does not appear to be impaired - patients automatically make predictive and prudential inferences on the basis of the whole body - the mechanisms associated with linking self-attribution to bodily representation, instead, appear to be affected.

What are the pertinent neural correlates? Among recently proposed models, decisional determinants are cast as complementary features of control and motivation, neurophysiologically distinguished as mutually reciprocal operations of the dorsolateral and medioventral frontal cortical regions [36]. These two operations together are hypothesized to comprise drive to goal actions, understood as intentional, but embracing motivational variation. As proposed, decisional effectiveness is elicited through parcellation of conditional independencies that are hierarchically distributed, e.g., learned vs hardwired, to create probabilistic generative models servicing maximal self preservation (energetically) Cast in this formulation, decisional outcomes bind self to body via goals in probabilistic action sequences. The role of choice in predictive inferencing is thereby 'eluded', restricted to inverting a generative model to infer an action sequence [36]. Saliency is then understood to be decisional through incentivization, rather than orthogonal to executive control, where correspondence to high prior probability has high motivational value. This interpretation suggests that decisional events emerge from learned, randomly evoked variation in prewired contingencies that are linked to saliency mechanisms that are constrained by generative, predictive modeling.

However, saliency can be understood, instead, as inductive, instilling repetition, and conferring precision. Goal progression, for example, increases its anticipated likelihood of attainment. When precision is inferred, successful goal directed behavior creates positive feedback 
[37]. This is to say that saliency induces a form of meta-learning, linking outcomes precisely with action, rather than with probabilistic inferencing. Decisional enactments can be expected to increasingly draw from meta-learning paradigms that reduce uncertainty and improve consequence. By inferring precision, goal directed behavior confers a salience on predictive reliability that overcomes probabilistic modes linked to free energy exigencies and creates the primitive circumstances for decisions based on causal conclusions, that is, the primitive for rational behavior. Taken together, schizophrenia aberrations are consistent with this latter interpretation and reveal that predictive assessments are not the sole form of inferencing. By lesioning the junction between bodily source and goal, they also reveal that inferencing is likely to be staged, at lower probability levels and higher, rationally deductive ones.

\section{Conclusions}

Disturbances in the sense of self that mark schizophrenia in prodromal and acute stages and current neural models of selfidentification suggest that the loss of self as a core symptom involves whole body representation. Based on evidence from schizophrenia patients there exist two levels of self-recognition, one automatic for action identification, the second, conscious and employed for conducting intentional actions. This latter determinant, altered in schizophrenia, is likely to entail rational primitives that bind self to goal through deductive inferencing.

\section{References}

1. Hur JW, Kwon JS, Lee TY, Park S (2014) The crisis of minimal self-awareness in schizophrenia: a meta-analytic review. Schizophr Res 152: 58-64. [Crossref]

2. Hecht D (2010) Schizophrenia, the sense of 'self' and the right cerebral hemisphere. Med Hypotheses 74: 186-188. [Crossref]

3. Wagner NF, Northoff G (2014) Habits: bridging the gap between personhood and personal identity. Front Hum Neurosci 8: 330. [Crossref]

4. Jeannerod M (2005) Levels of representation of goal-directed actions. In: HJ Fruend, M Jeannerod, M Hallett, R Leiguarda, eds. Higher-order Motor Disorders. Oxford: Oxford University Press.

5. Smith L (2009) Stability and flexibility in development. In: J Spencer, MSC Thomas, JL McClelland, eds. Toward a unified theory of development. Oxford: Oxford University Press.

6. Brincker M (2014) Navigating beyond "here \& now" affordances-on sensorimotor maturation and "false belief" performance. Front Psychol 5: 1433. [Crossref]

7. Noel JP, Pfeiffer C, Blanke O, Serino A (2015) Peripersonal space as the space of the bodily self. Cognition 144: 49-57. [Crossref]

8. Tallon-Baudry C, Campana F, Park HD, Babo-Rebelo M (2018) The neural monitoring of visceral inputs, rather than attention, accounts for first-person perspective in conscious vision. Cortex 102: 139-149. [Crossref]

9. Jeannerod M (2009) The sense of agency and its disturbances in schizophrenia: a reappraisal. Exp Brain Res 192: 527-532. [Crossref]

10. Lashley KS (1951) The problem of serial order in behavior. In: LA Jeffress, ed. Cerebral mechanisms and behavior. New York: Wiley Press 112-136.

11. HELD R (1961) Exposure-history as a factor in maintaining stability of perception and coordination. J Nerv Ment Dis 132: 26-32. [Crossref]

12. Frak V, Nazir T, Goyette M, Cohen H, Jeannerod M (2010) Grip force is part of the semantic representation of manual action verbs. PLoS One 5: 9728. [Crossref]

13. Thelen E, Smith L (1994) Dynamic systems approach to the development of cognition and action. Cambridge, MA: MIT Press.
14. Von Holst E, Mittelstaedt H (1950) Das reafferenzprinzip. Wechselwirkungen zwischen Zentralnervensystem und Peripherie. Naturwissenschaften. 37:464-476.

15. Weigmann K (2013) Our sense of self. Phenomenology is a philosophical discipline that gives a detailed description of selfhood; it can contribute to understanding psychiatric diseases such as schizophrenia and its neurological causes. EMBO Rep 14: 765-768. [Crossref]

16. Thelen E, Smith L (2005) Dynamic systems theories. http://www.iub.edu/ cogdev/ labwork/handbook.pdf

17. Shapiro L (2011) Embodied cognition. New York: Routledge Publishing.

18. Campos JJ, Bertenthal BI, Kermoian R (1992) Early experience and emotional development: the emergence of wariness of heights. Psychol Sci 3:61-64.

19. Bushnell EW, Boudreau JP (1993) Motor development and the mind: the potential role of motor abilities as a determinant of aspects of perceptual development. Child Dev 64: 1005-1021. [Crossref]

20. Lockman JJ, Thelen E (1993) Developmental biodynamics: brain, body, behavior connections. Child Dev 64: 953-959. [Crossref]

21. Gallese Gallese V (2000) The acting subject: toward the neural basis of social cognition In: Metzinger T, ed. Neural correlates of consciousness: empirical and conceptual questions. Cambridge, MA: MIT Press, 325-333.

22. Damasio A (2012) Self Comes to Mind: Constructing the Conscious Brain. New York: Pantheon Books.

23. Carroll J (2012) The truth about fiction: biological reality and imaginary lives. Style 46: $129-160$.

24. Seth AK, Suzuki K, Critchley HD (2012) An interoceptive predictive coding model of conscious presence. Frontiers Psychol 2.

25. Northoff G (2008) Are our emotional feelings relations? a neurophilosophical investigation of the James-Lange theory. Phenom Cog Sci 7: 501-527.

26. Frith C (2012) Explaining delusions of control: the comparator model 20 years on Conscious Cogn 21: 52-54. [Crossref]

27. Frith CD, Blakemore S, Wolpert DM (2000) Explaining the symptoms of schizophrenia: abnormalities in the awareness of action. Brain Res Brain Res Rev 31: 357-363. [Crossref]

28. Allen M, Friston KJ (2018) From cognitivism to autopoiesis: towards a computational framework for the embodied mind. Synthese 195: 2459-2482. [Crossref]

29. Parnas J, Handest P, Jansson L, Vollmer-Larsen A, Saebye D (2011) Self experience in the early stages of schizophrenia: 5 year followup of the Copenhagen Prodromal Study. World Psychiatry, 10(3):200-204.

30. Stanghellini G1 (2009) Embodiment and schizophrenia. World Psychiatry 8: 56-59. [Crossref]

31. Bloch A, Lambiotte R, Roberts B, Giedd J, Gogtay N, et al. (2012) The discovery of population differences in network community structure: new methods and applications to brain functional networks in schizophrenia. NeuroImage 59: 3889-3900.

32. Decety J, Sommerville JA (2003) Shared representations between self and other: a social cognitive neuroscience view. Trends Cogn Sci 7: 527-533. [Crossref]

33. Daprati E, Sirigu A, Pradat-Diehl P, Franck N, Jeannerod M (2000) Recognition of self produced movement in a case of severe neglect. Neurocase 6: 477-486.

34. Feinberg I (1978) Efference copy and corollary discharge: implications for thinking and its disorders. Schizophr Bull 4: 636-640. [Crossref]

35. BayneT, Pacherie E (2007) Narrators and comparators: the architecture of agentive self-awareness. Synthese 159: 475-491.

36. Pezzulo G, Rigoli F, Friston KJ (2018) Hierarchical Active Inference: A Theory of Motivated Control. Trends Cogn Sci 22: 294-306. [Crossref]

37. D'Angelo E, Casali S (2013) Seeking a unified framework for cerebellar funtion and dysfunction: from circuit operations to cognition. Front Neural Circuits. 6: 1-23. [Crossref]

Copyright: (C2018 Larrivee D. This is an open-access article distributed under the terms of the Creative Commons Attribution License, which permits unrestricted use, distribution, and reproduction in any medium, provided the original author and source are credited. 